\title{
Lire dans une langue étrangère (L2) à l'école: quelles stratégies de lecture pour quel genre de texte?
}

\section{Marianne Jacquin}

Dans cet article, nous nous intéressons à la lecture scolaire dans une langue étrangère (L2) et plus particulièrement à l'influence du genre de texte sur le choix de stratégies de lecture mobilisées par des élèves de $9^{e}$ année du Cycle d'Orientation genevois en classe d'allemand. Le dispositif didactique que nous avons mis en place nous a permis de saisir le processus de comprébension de textes, tel qu'il se construit au sein des interactions entre élèves, dans sa dimension dynamique et contextualisée par le biais de tâches à résoudre sur deux genres de textes contrastés (interview et récit narratif). Nous avons pu mettre en évidence des focalisations particulières et des trajectoires de lecture divergentes selon le genre abordé. Nous faisons l'hypothèse que ces résultats s'expliquent par le fonctionnement du genre respectif, instaurant des types de coopération avec le texte de nature différente (Eco, 1985; Ricoeur, 1983).

\section{Introduction}

Les modèles de lecture interactionnistes issus de la théorie cognitive des années 80 se caractérisent par une forte centration sur le pôle «lecteur» et la description des opérations internes que celui-ci met en œuvre pour comprendre un texte. Ces modèles postulent des savoirs, des processus et des stratégies universelles transférables d'une situation de lecture à une autre. Ils partent du point de vue qu'un lecteur en langue maternelle (L1) lit de la même manière qu'un lecteur en langue étrangère (L2), sauf que ce dernier rencontre davantage d'obstacles liés à une maîtrise linguistique moindre. Cette tendance à une uniformisation des processus de lecture en L1 et L2 touche aussi le pôle «texte». La dimension textuelle est évoquée en termes de schèmes formels - des savoirs à propos du genre et de la structure rhétorique du texte - activés par le lecteur (p. ex. Carrell, 1984) en même temps que des schèmes linguistiques (décodage, lecture mot par mot). L'idée sous-jacente qui traverse une grande partie de la recherche est celle d'une mobilisation de différents types de savoirs et savoir-faire qui viendraient soutenir la construction du sens d'un texte de manière identique, quel que soit le genre lu. 
L'approche d'enseignement par les stratégies de lecture et le courant plus récent de la didactique intégrée des langues illustrent, sur un plan didactique, cette position universaliste qui mise sur la transférabilité de savoirs acquis en L1 vers des situations d'apprentissage en L2. Les études menées - soit sur l'utilisation de stratégies de lecture par des lecteurs, soit sur les effets de l'enseignement de stratégies sur la capacité de comprendre des textes - se basent sur des textes de genres divers, mais ne considèrent que dans de rares cas la variable "genre de texte» comme jouant un rôle dans les processus de lecture.

La recherche que nous présentons dans cet article adopte une perspective différente. Menée dans un contexte scolaire, elle cherche à mettre en évidence la nécessité d'une prise en compte du genre textuel comme composante essentielle dans l'apprentissage de la lecture en L2.

L'article se compose de trois parties: une première partie qui expose le cadre théorique, une deuxième qui décrit la problématique, les questions de recherche, la méthodologie de recueil et d'analyse des données. La troisième partie présente quelques résultats sous forme d'une analyse contrastée de trajectoires de lecture (interview et récit narratif) par des élèves de $9^{\mathrm{e}}$ année du Cycle d'Orientation à Genève.

\section{Cadre théorique}

Les modèles de lecture issus de la psycholinguistique considèrent le texte comme une unité de sens fixe, donné une fois pour toutes. L'activité du lecteur consiste à tirer un certain nombre d'informations du texte afin d'en construire une représentation d'ensemble. Les théories herméneutiques, au contraire, envisagent le rapport du lecteur et du texte sous un angle plus dynamique, le sens du texte pouvant se transformer au fil de la lecture. ${ }^{1}$ D'après Eco (1985), le texte instaure un programme de lecture à travers le lecteur modèle conçu par l'auteur. C'est la coopération du lecteur qui permet d'actualiser le texte. «Les espaces blancs, les [...] interstices» de textes plus ou moins 'résistants' sont comblés par le lecteur, qui contribue ainsi à l'élaboration progressive du sens, toujours en mouvement, des textes (Eco, 1985, pp. 62-63). Pour un lecteur-apprenant en L2 s'ajoutent des obstacles liés à la langue, ce qui transforme les formes possibles de coopération avec le texte et, plus particulièrement, le rôle que peuvent jouer les savoirs sur les genres textuels.

De la double résistance des textes pour la lecture en L2 Nous reprenons ici l'idée de Vandendorpe (1992) que la quête du sens se met en route par la résistance qu'oppose le texte au lecteur. Dans une situation de lecture en L2, le texte résiste doublement, dans la mesure où il contient à la fois des lacunes volontairement introduites par l'auteur et des zones d'ombre dues à des la- 
cunes d'ordre linguistique du côté du lecteur, qui font obstacle à une compréhension plus littérale, locale d'un passage. ${ }^{2}$ La recherche du sens ou d'une cohérence du texte est dès lors ancrée dans des îlots de textes qui deviennent des points de départ mettant en mouvement la démarche interprétative. Celle-ci vise à combler simultanément les lacunes des deux types. Si l'interprétation devient, dans certain cas, un outil à la compréhension (Tauveron, 2004), à d'autres moments, la contrainte inhérente à la lecture L2, qui est celle de ne pouvoir choisir le point de départ de son activité interprétative, peut conduire à la création d'un monde différent ou décalé par rapport à celui du texte ${ }^{3}$, voire à une rupture de coopération avec le texte.

Les textes résistent aussi par «des éléments potentiellement polysémiques, des indices pouvant entrer dans plusieurs réseaux et donc diversement interprétables» (Mainguenau, 1990, cité dans Tauveron, 1999, p. 21). Pour le cas de la lecture en L2, nous sommes à nouveau dans une double origine de la polysémie des éléments du texte. Celui-ci offre plusieurs interprétations possibles non seulement par son caractère "ouvert» voulu par l'auteur, mais aussi pour des raisons d'ordre linguistique. D'abord, le lecteur L2 isole les parties du texte comprises de leur co-texte, en créant des sortes de réseaux d'îlots de compréhension qui correspondent à autant d'interprétations possibles de l'ensemble. Ensuite, le lecteur rencontre des problèmes au niveau de la signification des unités lexicales ou syntagmatiques en jeu. Il hésite entre plusieurs traductions possibles. En l'absence d'une compréhension du co-texte, son choix est guidé soit par la décision locale d'attribution d'une signification au mot qu'il pense être la plus probable en fonction de ses connaissances, soit par la logique d'ensemble du texte basée sur les réseaux d'îlots de compréhension constitués auparavant.

Ces quelques considérations nous mènent vers une redéfinition de la notion de coopération adaptée à notre contexte.

\section{Quel type de coopération entre un texte et un lecteur L2?}

Les conditions particulières de la lecture en L2 telles que décrites ci-dessus modifient le type de coopération d'un lecteur L2 avec le texte de trois manières. La première est liée au fait qu'un lecteur en L2 est contraint de construire la cohérence globale d'un passage ou d'un texte à partir d'indices fragiles, peu consolidés. Le texte subit ainsi une sorte de distorsion, par le fait que le lecteur ne dispose que dans une certaine mesure de la possibilité de vérifier ses hypothèses de lecture.

Deuxièmement, le lecteur en L2 est essentiellement apprenant-lecteur. A l'école, le texte devient support d'apprentissage, ce qui oblige le lecteur à mobiliser l'ensemble de ses ressources disponibles, dans le but à la fois de créer une cohérence du texte et de construire de nouvelles connaissances linguistiques ou stratégiques.

Troisièmement, l'activité de lecture est fortement contextualisée. Elle se déroule dans un cadre didactique qui prévoit un genre de texte, accompagné de 
tâches de lecture formant une unité qui oriente la coopération avec le texte. A l'instar de l'auteur, l'enseignant conçoit un lecteur-apprenant modèle qui s'engagerait dans une tâche de lecture de telle sorte qu'elle lui permette simultanément d'accéder, hic et nunc, au sens du texte, à un degré de compréhension visée par la tâche, tout en faisant appel à l'ensemble des savoirs nécessaires pour accomplir celle-ci et, ainsi, améliorer, à plus long terme, sa performance de lecture. La coopération avec le texte s'inscrit ainsi dans une coopération définie par le contrat didactique. Mais, et c'est ce qui nous intéresse plus particulièrement ici, les contraintes structurelles d'un texte donné, et d'une manière plus générale, le fonctionnement du genre auquel ce texte appartient influent sur le parcours de lecture.

\section{De la pertinence de la notion de genre textuel pour la lecture en L2}

Le genre est considéré comme "un outil sémiotique complexe, c'est-à-dire une forme langagière prescriptive qui permet à la fois la production et la compréhension de textes» (Schneuwly, 1994, p. 160; notre soulignement). «Les genres [...] fonctionnent comme l'interface qui permet l'interaction entre celui qui produit et celui qui reçoit» (Schneuwly, 2007, p. 14; notre soulignement).

Lorsque le genre entre en classe, il fait l'objet d'une transposition didactique et devient genre scolaire (Schneuwly \& Dolz, 1997). Détaché de son contexte communicationnel ${ }^{4}$ d'origine, il n'est plus seulement outil de communication, mais en même temps objet d'enseignement-apprentissage permettant, p. ex. sous une forme simplifiée, aux élèves de s'approprier des capacités transférables à d'autres genres proches ou lointains.

La question qui se pose dans notre contexte est celle de savoir dans quelle mesure le genre peut être considéré comme objet et/ou outil d'apprentissage, visant le développement de la capacité de lecture en L2.

Dans l'approche interactionniste, la notion de mobilisation de schèmes formels tend vers une utilisation d'un savoir sur les genres comme un outil parmi d'autres, en quelque sorte «externe» ou préalable à la lecture. C'est le lecteur qui mobilise des savoirs sur le fonctionnement du genre en L1 pour comprendre un texte en L2, sans que le genre ne fasse l'objet d'un apprentissage en tant que tel. Si on considère, au contraire, que la construction du sens d'un texte ne peut pas se faire indépendamment du fonctionnement du genre auquel il appartient, on peut faire l'hypothèse que celui-ci déterminera les cheminements qu'emprunte le lecteur, indépendamment du degré de connaissances qu'il pourrait par ailleurs avoir sur un genre donné. Dans ce sens, un texte peut devenir, non seulement support d'apprentissage à la lecture L2 en général, mais outil d'apprentissage du genre en question. 


\section{Problématique et questions de recherche}

Une grande diversité de genres textuels est présente aussi bien dans les plans d'études, les manuels d'enseignement que dans la recherche. Mais cette diversité proposée aux enseignants et élèves ne s'accompagne pas d'un enseignement outillé de stratégies de lecture spécifiques aux différents genres. On fait en quelque sorte le pari que la confrontation de l'élève à différents genres de textes lui permettra de développer des stratégies adaptées.

La recherche, de son côté, se limite souvent à une description inventoriée de stratégies universellement efficaces, c'est-à-dire permettant d'aboutir à un résultat de compréhension satisfaisant, défini par ailleurs de manière décontextualisée.

Située dans un contexte didactique, notre recherche s'intéresse au processus de construction de la compréhension et du sens d'un texte donné, à travers les transformations successives du sens des parties du texte et de son ensemble, telles qu'on peut les observer grâce à des analyses de trajectoires de lecture ${ }^{5}$.

Les questions principales portant sur le rôle du genre textuel pour la lecture L2 sont les suivantes:

- Comment le genre textuel influe-t-il, en termes de stratégies utilisées et d'obstacles rencontrés, sur les trajectoires de lecture empruntées par les élèves?

- Quel type de cohérence textuelle les élèves construisent-ils selon le genre de texte?

Avant de répondre à ces questions par une analyse contrastée de parcours de lecture, nous donnons, dans la partie suivante, quelques indications sur la méthodologie de recueil et d'analyse des données.

\section{Méthodologie}

Les difficultés méthodologiques inhérentes à la description du processus de lecture échappant à l'observation directe ont maintes fois été évoquées dans la littérature (voir p. ex. Swain, 2006). Le concept de «stratégie de lecture» présentait pour nous deux avantages. Il nous a d'abord permis de disposer d'un objet d'enseignement clairement défini qui pouvait être proposé à des enseignants sous forme d'une séquence didactique. La manière dont les élèves mobilisent ces stratégies de lecture préalablement enseignées devient ensuite observable par le biais des interactions, médiatisées par la tâche de lecture. 


\section{Recueil et analyse des données}

La récolte des données s'est effectuée durant l'année scolaire 2004-2005 dans quatre classes de $9^{e}$ année (élèves de 15 à 16 ans) du Cycle d'Orientation à Genève. Les élèves en fin de scolarité obligatoire disposent d'une certaine expérience dans la lecture de l'allemand et ont été confrontés à une grande variété de genres de textes en L1. Pour pouvoir observer le déroulement on line du processus de lecture en classe, il était en effet plus intéressant de pouvoir proposer des textes d'une certaine longueur et complexité. Les élèves ont aussi eu l'occasion d'expérimenter différentes formes sociales de travail dont le travail en groupes, nécessaire à notre dispositif de recherche, qui vise à faire émerger des traces orales permettant d'appréhender, en temps réel, comment les lecteurs construisent la compréhension et le sens du texte (Giroud Krajewski, 2001, p. 57). A ces fins, nous avons conçu une séquence didactique composée de trois phases, mises en place par deux enseignantes volontaires. La première phase consistait à introduire l'objet d'enseignement «stratégies de lectures» (une période), et était suivie de deux phases de travaux de groupes sur la lecture d'une interview et d'un récit narratif ( $2 \times 2$ périodes). Les enregistrements vidéoscopiques ont eu lieu à trois moments dans l'année, respectivement en octobre, novembre 2004 et avril 2005.

Nous avons ensuite transcrit les parties significatives des interactions entre les élèves en lien avec nos questions de recherche. Après une condensation des données à l'aide de synopsis, nous avons conçu un outil d'analyse qui nous permet de reconstruire les parcours de compréhension des élèves à partir d'une mise en relation d'indices présents aussi bien dans leurs discours que dans les textes.

Le choix des textes, une interview menée avec un jeune qui parle de ses loisirs et un récit fictif de Franz Hohler a été guidé par des critères à la fois didactiques et en lien avec nos questions de recherche. D'un point de vue didactique, il fallait trouver des textes authentiques motivants pour les élèves, présentant un niveau linguistique légèrement supérieur à leur capacité de réception afin qu'une utilisation de stratégies de lecture s'avère nécessaire. Les textes ont en effet été sélectionnés pour permettre le développement d'une lecture stratégique en L2 à partir de deux genres dont la structure discursive était familière aux élèves. Du point de vue de la recherche, le fonctionnement contrasté et les contrats de lecture qu'instaurent respectivement chacun des genres nous semblaient pouvoir mettre en évidence des stratégies diversifiées.

\section{Le fonctionnement des genres textuels «interview» et "récit»: une analyse a priori}

Les deux genres «interview» et "récit narratif» retenus pour notre recherche présentent des fonctionnements différents, voire opposés en ce qui concerne les rapports qu'ils entretiennent entre leurs parties et le tout. Dans la situation communicative de l'interview, l'alternance entre questions et réponses et leur déroulement chronologique instaure, même en présence d'un fil rouge thématique dont l'interviewer est le garant, des ruptures entre les différentes parties du 
texte, présentées par sous-thèmes, de telle sorte que le récit des événements dans chaque partie se suffit en quelque sorte à lui-même. La cohérence devient ainsi davantage visible au niveau de chaque partie qu'à celui de l'ensemble de l'interview.

Le genre du récit de fiction fonctionne, à plusieurs égards, de manière opposée. Contrairement à l'interview, qui évoque le monde "réel» à travers le discours d'une personne, le récit raconte une histoire en créant un monde particulier à travers la construction d'un espace-temps, d'événements, d'actes et de personnages avec leurs paroles et pensées. - [...] Pour qu'il y ait récit, il faut d'abord la représentation d'une succession temporelle d'actions, il faut ensuite qu'une transformation plus ou moins importante de certaines propriétés initiales des actants soit réalisée ou échoue, il faut enfin qu'une mise en intrigue structure et donne sens à cette succession d'actions et d'événements dans le temps. (Adam, 1997, (Charaudeau \& Maingueneau, 2002, p. 484; notre soulignement)

C'est à partir des notions de mise en intrigue et de reconfiguration que $\mathrm{Ri}$ cœur (1984) évoque la notion de cohérence textuelle globale du récit. L'intrigue dit-il,

fait médiation entre des événements ou des incidents individuels et une histoire prise comme un tout. [...] En conséquence, un événement doit être plus qu'une occurrence singulière. Il reçoit sa définition de sa contribution au développement de l'intrigue. Une histoire, d'autre part, doit être plus qu'une énumération d'événements dans un ordre sériel, elle doit les organiser dans une totalité intelligible. De telle sorte qu'on puisse toujours demander ce qu'est le 'thème' de l'histoire. Bref, la mise en intrigue est l'opération qui tire d'une simple succession une configuration. (Ricoeur, 1994, p. 127; notre soulignement)

A ces différences fondamentales dans les conditions de production des textes correspondent des modalités de réceptions qui different d'un genre à l'autre et qui ont des conséquences sur les parcours de lecture. Le lecteur d'une interview peut faire le choix de ne lire qu'une partie de l'entretien. Même si les parties entretiennent des liens thématiques entre elles, leur organisation interne n'oblige pas contrairement au récit - à construire le sens global de l'interview ni à en comprendre la visée générale. Il n'en est pas de même pour le récit, où le sens de l'ensemble ne saurait être appréhendé sur la base d'une partie seulement. La (re)configuration opérée par l'intrigue, la transformation d'une succession d'événements en une configuration, loi du genre par excellence, impose un contrat de lecture qui oblige le lecteur à un parcours qui tienne compte des parties pour comprendre le tout et inversement. «Comprendre un récit [...] ce n’est pas décoder une à une des phrases et des phases de l'intrigue, c'est passer d'une successivité à un tout de sens cohésif-cohérent ressenti comme formant un texte» (Ricœur, 1984, p. 127). Parce que, continue l'auteur, «le récit est toujours plus qu'une série chronologique d'événements et d'actions, la lecture-compréhension d'un texte est un jugement réflexif́ ${ }^{6}$ qui (re)configure le texte. Elle est un acte qui comprend - qui 'prend ensemble' - le divers de l'action dans l'unité de l'intrigue.» 
A partir de ces quelques considérations sur les «contrats» de lecture différents instaurés par les deux genres, on peut faire l'hypothèse que ceux-ci influent sur le type de cohérence recherchée par le lecteur. Nécessairement globale pour le récit, on peut supposer qu'elle sera plus locale ou partielle pour l'interview. C'est ce que nous cherchons à montrer à travers l'analyse contrastée des trajectoires de lecture dans la partie suivante.

\section{Résultats}

\section{Lire une interview: la construction d'une cohérence partielle}

Dans le cadre de la lecture d'une interview sur les habitudes de télévision d'un jeune, les élèves répondent à une série de questions sur le texte, dont celle-ci: La télévision est-elle importante pour J.? (Justifie).

Voici l'extrait de l'interview sur lequel les élèves travaillent pour trouver la réponse. L'analyse a priori révèle un certain nombre d'obstacles potentiels sur le plan des savoirs linguistiques (en italique dans le texte). Au-delà de ce premier niveau de résistance qu'oppose le texte, on peut mettre en évidence les hésitations, nuances, implicites, voire les contradictions dans le discours de l'interviewé qui rendent la réponse à la question du rôle que joue la télévision difficile.

\section{Fernsehen: Videoabende Julian, 18 Jahre, Schüler, Berlin}

- Sitzt du viel vor dem Fernseher?

- Ich gucke eigentlich gar nicht so viel Fernsehen (...).

- Wie wichtig ist Fernsehen oder Video für dich?

- Es ist schon wichtig. Weil, es macht schon... Was ich manchmal noch mache - obwobl es eigentlich mehr so für Kinder ist - aber, ein Rollenspiel spielen. Das ist manchmal auch ganz lustig. Und Fernsehen gibt schon so 'nen gewissen Ansporn... Man sieht schon im Fernsehen viel, was man... wo man sich «selber» so in Situationen sieht, [structure phrase] wo man sich selber ein bisschen vielleicht, na ja, wiedererkennt und dann halt 'ne gewisse Handlungsmöglichkeit $d a$ drin gewinnt.

Handlungsmöglichkeit = etwas machen können

Mais regardons d'abord de plus près de quelle manière les élèves procèdent. Dans un premier temps, ils repèrent, à l'aide du mot-clé wichtig (important) le passage du texte susceptible de contenir des éléments de réponse à la question posée. Ils investissent l'extrait du texte essentiellement à travers des stratégies combinées des mots-clés, (en gras dans le texte), de la formulation d'hypothèses et des liens interprétatifs entre différents passages. La répétition du mot-clé wichtig semble inciter à l'hypothèse d'une grande importance de la télévision pour la personne interviewée. L'extrait suivant des interactions entre élèves illustre la recherche d'indices venant appuyer et justifier cette première hypothèse. 


\section{$\underline{\text { Extrait } 1}$}

13'33 Yor: oui: / attends il faut juste essayer de trouver dans le texte quelque chose

Tov: ben il dit es ist schon wichtig es ist schon wichtig parce que elle fait juste je sais pas quoi

Yor: mais si ça se trouve c'est pas important

Tov: manchmal ça veut dire quoi déjà

Yor: c'est parfois

Tov: ah oui parce que c'est parfois aussi ganz lustig parce que c'est aussi drôle //

14 '01 et puis Rollenspiel ça veut dire quoi

Yor: mais en haut c'est marqué passes-tu beaucoup de temps devant la télé et puis après c'est marqué je regarde volontiers gar nicht so viel mais je sais pas ce que ça veut dire je sais pas si c'est pas beaucoup

Tov: où tu vois gar nicht so viel

Yor: (pointe sur le texte à Tov) en haut // là

Tov: ah oui gar nicht so viel fernsehen je vois

Le subordonnant weil (parce que) combiné au mot-clé viel, caractérisant l'interviewé comme grand amateur du petit écran, vient corroborer la première hypothèse. C'est probablement une des raisons pour laquelle les élèves ne tiennent pas compte de la négation (nicht viel) qui apparaît dans la réponse. L'étayage argumentatif se construit également à partir de mots-clés, mis en réseau: weil manchmal - lustig (parce que - parfois - drôle).

Après une brève discussion sur le sens du mot inconnu eigentlich, les élèves reprennent la recherche d'arguments ce qui donne lieu aux échanges suivants:

\section{Extrait 2}

15’00 Tov: mais je veux dire oui c'est important il faut justifier il dit que / il dit que c'est important il dit que c'est souvent marra:nt et que: gib[t] schon so ne gewisse gewissen [Ansporn] ça veut dire quoi déjà

Yor: gewissen / wissen c'est savoir non c'est

Tov: oui wissen c'est savoir

Yor: c'est a su

Tov: c'est su ouais / gib gibt c'est geben es gibt non il y a es gibt c'est il y a (...)

15’31 Fernsehen gibt schon là (pointe la phrase dans son texte pour montrer à Yor) / im

Fernsehen gibt schon il y a schon so nen gewissen [Ansporn]

Yor: ah oui

Tov: ils savent beaucoup de choses

Yor: oui

Tov: il y a beaucoup de choses qu'ils peuvent (?) apprendre

Un lien est opéré entre deux unités lexicales gewissen (certain) et gibt (donne) sans que celles-ci ne soient soumises à une analyse grammaticale. C'est ainsi que gewissen (certain) est assimilé au verbe wissen (savoir) et gibt (donne), associé à l'expression es gibt (il y a), ce qui transforme la télévision en source de savoir et en moyen pour apprendre. A l'argument du plaisir s'ajoute celui de son utilité. 
On notera au passage que l'adverbe manchmal, traduit correctement dans l'extrait 1 est transformé ici en "souvent», soulignant ainsi davantage l'idée centrale de départ. On constate la force qu'acquiert peu à peu la logique construite autour des superlatifs (très, beaucoup, souvent), remettant en cause un savoir linguistique pourtant bien présent.

L'échange se termine par la mise en évidence du mot-clé Handlungsmöglichkeit (possibilité d'agir) qui fournit, à partir d'une généralisation, le dernier argument: avec la télévision, tout est possible. Les élèves concluent en utilisant la forme du texte - en occurrence la longueur de la réponse - comme indice supplémentaire de la grande importance que doit avoir la télévision dans la vie de la personne.

Cette brève analyse d'une étape de la trajectoire montre une tendance générale à partir d'éléments connus, au niveau lexical, sur la base desquels sont émises des hypothèses. La cohérence d'un passage du texte se construit, au fil de la lecture, par le biais d'une mise en réseau de ces mots-clés choisis en fonction de leur apport pour alimenter les hypothèses de départ. On voit bien aussi que les élèves ne tirent pas (suffisamment) profit de leurs connaissances lexicales et ne mobilisent pas les savoirs grammaticaux acquis pendant leur scolarité. La seule connaissance du sens d'un mot ne semble pas suffisante pour mobiliser ce savoir dans le contexte de la lecture, les élèves font davantage confiance à leurs premières hypothèses qu'à leurs savoirs linguistiques qui pourraient remettre celles-ci en question. On assiste à une forme de régression dans le parcours de compréhension. Tout se passe comme si, une fois quelques éléments du texte repérés, la construction de la logique ne pouvait se faire que dans un mouvement descendant et linéaire, écartant d'une part tout élément qui viendrait la remettre en question, mais en puisant, d'autre part, de manière sélective, des indices permettant de l'alimenter.

Cette primauté de la cohérence textuelle, qui, dans le cas du genre interview correspond à une cohérence partielle ${ }^{7}$, nous y reviendrons, permet de faire le constat que le premier niveau de résistance opposé par le texte - celui concernant d'hypothétiques lacunes linguistiques en L2 - ne peut pas, à lui seul, justifier la manière de procéder des élèves. La source de l'obstacle réside probablement davantage dans une difficulté à comprendre les traces de l'oralité, spécifiques au genre, à savoir les hésitations et nuances apportées par des adverbes, occultant ainsi des mots connus par les élèves (p. ex.: nicht dans eigentlich gar nicht so viel).

Cependant, si les élèves réussissent, malgré des obstacles locaux, à comprendre certaines parties du texte liées strictement à la tâche, c'est parce que le fonctionnement du genre le permet. Celui-ci autorise à naviguer exclusivement dans certaines parties du texte, à la recherche d'informations ciblées permettant de construire une cohérence partielle, interne à un passage donné. Même si les liens créés entre des îlots de compréhension isolés posent certains problèmes au niveau d'une compréhension plus fine comme nous venons de le montrer, ce procédé n'entraîne pas de conséquences au niveau de la compréhension de l'ensemble. La 
situation de lecture d'un récit au contraire, impose des trajectoires d'un tout autre type, comme nous allons le développer dans la partie suivante.

\section{Lire un récit narratif: la construction d'une cohérence globale}

La lecture d'un récit narratif incite les élèves à recourir davantage à des stratégies de lecture textuelles (comme émettre des hypothèses, poser des questions au texte, faire appel aux connaissances sur le genre, ...). Une des stratégies dominantes consiste à relier entre eux différents éléments compris du texte. Cette démarche s'appuie soit sur des mots-clés textuels, soit sur des îlots de signification plus larges, formés par des phrases traduites intégralement en langue 1. Lapparition de ces données quantitatives nouvelles, la nature des obstacles rencontrés par les élèves ainsi qu'une manière différente de construire le sens du texte que nous allons illustrer dans l'exemple qui suit, laissent à penser qu'elles sont directement liées aux caractéristiques du genre récit, à la narrativité du texte.

Dans le cadre d'une tâche de texte puzzle ${ }^{8}$ sur un récit de P. Maar, Der Mann, der nie zu spät kam, les élèves sont amenés à retrouver l'ordre des extraits dont ils disposent et à émettre des hypothèses sur les parties du texte manquantes. Le texte met en scène un personnage obsédé par la ponctualité. Le retard d'un train qui lui sauve la vie lui fait prendre conscience de l'existence d'autres valeurs.

Lanalyse du parcours de lecture des élèves, dont nous décrivons les principales étapes, montre de quelle manière ils se servent de leurs savoirs liés au schéma narratif classique pour restituer l'ordre des extraits.

La lecture conjointe de la première phrase de l'extrait 4 (Wilfried verabredete sich nie mit anderen, hatte nie Termine und ging nie aus, weil er Angst hatte, zu spät zu kommen) et du titre (Der Mann, der nie zu spät kam) permet d'emblée de définir le thème en termes de tension entre ponctualité et retard, opposition qui fonctionne comme guide tout au long de la lecture. Ce fil rouge permet, sur la base du mot-clé spät de créer des liens entre les différents extraits. Combiné à la mobilisation d'un savoir concernant la structure générale du récit, plus particulièrement l'idée selon laquelle un récit se termine «bien», la stratégie aide à (re)structurer le récit en ordonnant les extraits selon une première logique, allant d'une situation initiale (titre), exempte de problèmes, vers un premier classement des extraits 4 et 6 parlant respectivement de l'arrivée ponctuelle du personnage et d'aspects positifs à la fin. ${ }^{9}$ (Ich habe inzwischen nur verstanden, dass Verspätungen auch sehr positiv sein können.)

Ces premières hypothèses quant à la chronologie des extraits entraînent, dans un deuxième temps, la lecture vers une logique de classements d'extraits en deux catégories, ceux qui racontent un retard du personnage (négatif) et ceux où il est à l'heure (positif). Ce procédé influe sur le choix des unités du texte à investir et sur la compréhension à un niveau plus local. Les interactions des élèves portant sur l'extrait 2 (description de l'enfance du personnage) sont, de ce point de vue, significatifs. 
Extrait 3

21'56 Tov: mais LÀ rega:rde moi je trouve que c'est vraiment une conclusion quand tu dis rannte sofort nach [nach Hause] je n'étais pas en retard ni à manger ni pour arriver ni pour venir je sais pas euh quand le Hausmeister le chef de maison je sais pas quoi

(8") et tous les autres enfa:nts ils jouaie:nt tout près de l'école au foot e:t ça c'est la FIN ça c'est la fin je suis sûre regArde il raconte tout ce qui se passe autour de lui il dit que ouai::s les petits enfants ils jouent

22'31 encore au football à côté de la maison il les regardait et tout et puis que ça rendait heureux et das tat

Yor: oui ça doit être la fin /

La phrase finale de l'extrait (Er rannte sofort nach Hause, um nicht zu spät zum Essen zu kommen) qui sert d'illustration au trait de caractère pathologique déjà présent dans l'enfance, et fait donc partie du début de l'histoire, est interprété par les élèves comme une possible "conclusion", par le simple fait qu'elle peut être classée comme extrait "positif» à partir de mots-clés (nicht zu spät). Afin de vérifier leur hypothèse, les élèves cherchent des indices dans une autre phrase, facilement accessible par le biais de mots-clé connus (Andere Kinder spielten nach der Schule Fussball, und schauten sich auf dem Heimweg die Schaufenster an). Ils s'en servent pour généraliser, par le biais d'un résumé, l'ensemble du paragraphe («Il raconte ce qui se passe autour de lui») et comme point de départ d'une interprétation allant dans le sens de la logique du schéma narratif déjà évoqué. Ils érigent la scène des enfants jouant au football comme symbole d'un monde heureux («Il raconte ce qui se passe autour de lui et ça le rendait heureux») justifiant ainsi son emplacement à la fin du récit.

Si on peut rencontrer des stratégies similaires, notamment des hypothèses effectuées à partir de mots-clés, le genre narratif contraint plus fortement à effectuer des liens entre les différentes parties du texte. ${ }^{10}$ Contrairement à la lecture de l'interview autorisant une compréhension partielle guidée par un projet de lecture unique, la construction du sens du récit procède par étapes, à travers un enchaînement de projets de lecture successifs, qui passent d'un classement des extraits en fonction de la thématique générale, à un aspect du schéma narratif (la fin heureuse) vers la prise en compte de l'ensemble de la structure narrative classique. On voit bien aussi que l'obstacle rencontré ne se situe plus essentiellement au niveau d'un passage donné, mais empêche véritablement l'accès au sens de l'ensemble, jusque dans la capacité à ordonner le récit chronologiquement à l'aide d'indices textuels. Les incohérences perçues au niveau de la structure d'ensemble obligent à un retour sur le texte, à une lecture de passages qui n'avaient pas été pris en considération lors de la première lecture. On observe aussi une transformation dans la mise en œuvre des stratégies qui ne se cantonne plus à un passage donné visant une compréhension partielle à l'aide d'un mouvement unidirectionnel, mais cherche à construire une logique d'ensemble, logique qui se consolide dans un mouvement circulaire, où les hypothèses et interprétations font partie intégrante de la construction en cours. 


\section{Conclusion: Vers une prise en compte du genre comme composante essentielle du contexte didactique en L2}

Un certain nombre de points communs entre la lecture d'une interview ou d'un récit sont observables en ce qui concerne la mise en œuvre de certaines configurations typiques de stratégies, la faible mobilisation de savoirs linguistiques L2 ou encore la primauté de la cohérence textuelle construite sur la base de quelques indices du texte. D’une manière générale, les élèves ne confrontent pas leurs premières hypothèses à l'aide d'une relecture plus détaillée du texte.

Cependant, la manière de mettre en ouvre les stratégies, la nature des obstacles et leur effet sur la compréhension à différents niveaux, ainsi que la présence ou absence d'une mobilisation de savoirs sur le genre sont autant d'illustrations des différences et démontrent les limites du transfert de stratégies de lecture «universelles» d'un genre à l'autre.

Les stratégies mobilisées pendant la lecture de l'interview - genre qui autorise une lecture partielle, voire fragmentée - remplissent leur rôle, dans la mesure où elles permettent d'atteindre une compréhension suffisante dans le contexte de la tâche à remplir. L'obstacle se situant à un niveau local, les implicites liés au discours des personnes interviewées et qui par ailleurs s'expriment à travers des formes linguistiques difficiles d'accès pour les élèves (traces de l'oralité), n'affecte pas la compréhension de l'ensemble. C'est peut-être une des raisons pour lesquelles il n'y a pas de traces, dans les interactions entre élèves, d'un savoir mobilisé sur le genre.

L'apparition d'une stratégie interprétative par mise en relation d'éléments dans l'ensemble du texte et la mobilisation des savoirs macro structurels sur le genre, fonctionnant comme "outil» (Schneuwly, 1994), et aidant à organiser et guider l'ensemble du parcours de lecture, sont des indices forts de l'influence du genre sur le processus de lecture. Le contrat de lecture impliquant une mise en relation (chrono) logique des parties, constitutives du tout entraîne des obstacles au niveau non plus d'un passage donné, mais de la construction du sens global.

Ces résultats doivent cependant être relativisés pour au moins deux raisons d'ordre méthodologique. La première a trait à l'observabilité des stratégies de lecture mise en œuvre. Même si nos outils d'analyse permettent de retracer les parcours de lecture effectués par les élèves, ces derniers ne verbalisent que peu ce qu'ils mettent en œuvre. La deuxième raison est liée à la séquence didactique qui propose des tâches de types différents pour les deux genres de textes. On ne peut faire l'impasse sur le rôle important que joue la tâche de lecture (Jacquin, sous presse) dans ce contexte.

Nous soutenons néanmoins que les différences mises en évidence dans cet article peuvent avoir des implications didactiques. Si l'approche par stratégies de lecture permet certes de faire un premier pas dans la direction d'un enseignement de la lecture en tant qu'objet d'enseignement autonome, il s'agirait néanmoins 
de concevoir des dispositifs didactiques qui permettent d'intégrer un travail sur les caractéristiques du genre et une métaréflexion sur les conséquences à tirer pour la lecture en L2, p. ex. en termes d'(in)efficacité de certaines stratégies «universelles», telles que mots-clés connus, hypothèses. Cette optique aurait l'avantage de se pencher sur la question d'un enseignement spécifique de la lecture en L2, dans des genres donnés.

\section{Notes}

1 Dans la perspective de l'apprentissage de la lecture en L2, nous nous intéresserons moins au produit de la compréhension qu'au processus de construction au fur et à mesure de l'avancement dans le texte, à la transformation du sens des parties du texte et de son ensemble lors de la trajectoire ou, autrement dit au parcours interprétatif.

2 On peut attribuer ces deux niveaux à l'interprétation 1 et 2 chez Tauveron (1999). Les «blancs» du texte peuvent par ailleurs être considérés de nature différente selon le genre du texte. Dans le récit narratif, c'est le sens global qui résiste surtout à la compréhension alors que le genre «interview», de par son caractère oral et linéaire, contient plutôt des ellipses dans le discours des interviewés, des implicites liés à la situation de communication particulière.

3 Le décalage ici ne provient pas du fait qu'un lecteur procéderait à des hypothèses tous azimuts qui seraient complètement détachées du texte. Au contraire, c'est en quelque sorte le texte lui-même, tel qu’il se présente à lui, qui «guide» la construction d'une cohérence possible.

4 Un texte authentique s'adresse en premier lieu à des lecteurs natifs. Il peut ensuite faire l'objet d'une lecture par des lecteurs non natifs qui construisent des projets de lecture différents selon leurs besoins particuliers et le contexte scolaire ou extrascolaire. Dans le contexte scolaire, les lecteurs-apprenants L2 s'empareront de ce texte d'une manière différente que le lecteur L2 à l'extérieur de l'école, puisque leur projet de lecture sera déterminé par le dispositif d'apprentissage mis en place par l'enseignant. Les conditions de réception d'un genre de textes en L2 ne peuvent par conséquent pas être celles d'une situation de communication authentique.

5 Par «trajectoire de lecture», nous entendons le cheminement du lecteur dans le texte, son parcours chronologique d'un passage du texte à un autre, incluant les relectures.

6 Ricœur partage avec Mink (1965) la conception «narrativiste» «selon [laquelle] les récits sont des totalités hautement organisées, exigeant un acte spécifique de compréhension, de la nature du jugement. (...) caractère synthétique de l'activité narrative» (Ricœur, 1984, p. 276).

7 Il s'agit ici aussi d'un effet de la tâche qui oriente le lecteur vers une partie précise du texte.

8 Les deux dyades travaillent sur des extraits différents et complémentaires du texte, qui leur sont présentés dans le désordre.

9 Si cet extrait se situe effectivement à la fin, les raisons pour lesquelles c'est le cas échappent aux élèves.

10 On peut ici aussi parler d'un effet tâche (cf. note 7) dans la mesure où la recherche de la chronologie de l'histoire à partir d'extraits de textes mélangés oblige à adopter un point de vue plus global. 


\section{Bibliographie}

Adam, J.-M. (1997). Le style dans la langue. Une reconception de la stylistique. Lausanne: Delachaux et Niestlé.

Carrell, P. L. (1984). The effects of rhetorical organization on ESL readers. Tesol Quarterly, 18, (3), 441-469.

Charaudeau, P. \& Maingueneau, D. (2002). Dictionnaire d'analyse du discours. Paris: Seuil.

Eco, U. (1985). Lector in fabula. Le rôle du lecteur ou la coopération interprétative dans les textes narratifs. Paris: Grasset.

Giroud Krajewski, A. (2001). Au fil de l'écriture: étude du processus de production de textes argumentatifs en français langue étrangère à travers l'écriture à deux. Thèse de doctorat en Sciences de l'éducation, Université de Genève.

Jacquin, M. (2010). Enseigner et apprendre la lecture en allemand langue étrangère. La construction du sens des textes par les élèves. Sarrebruck: Editions Universitaires européens.

Jacquin, M. (2008). Enseigner la lecture en allemand en langue étrangère (L2) à l'aide un dispositif d'apprentissage coopératif: comment le sens d'un texte se construit-il dans l'interaction? Babylonia, 3, 36-42.

Jacquin, M. (sous presse). Strategisches Lesen im DAF-Unterricht: Welche Rolle spielt die Aufgabenstellung? Deutsch als Fremdsprache.

Maingueneau, D. (1990). Pragmatique pour le discours littéraire. Paris: Dunod, Bordas.

Mink, L.O. (1965). The Autonomy of Historical Understanding, History and Theory 5 (1), 24-47.

Ricœur, P. (1984). Temps et récit. Tome II. La configuration dans le récit de fiction. Paris: Seuil.

Schneuwly, B. (1994). Genres et types de discours: considérations psychologiques et ontogénétiques. In Y. Reuter (Éd.), Les interactions lecture-écriture: Actes du Colloque ThéodileCredil (Lille, novembre 1993) (pp. 155-173). Berne: Peter Lang.

Schneuwly, B. (2007). Genres écrits et oraux et forme scolaire. Enseignement et apprentissage de la langue première à l'école. In C. Boré (Éd.), Construire et exploiter des corpus de genres scolaires (pp. 13-26). Namur: Presses Universitaires.

Schneuwly, B. \& Dolz, J. (1997). Les genres scolaires: des pratiques langagières aux objets d'enseignement. Repères, 15, 27-40.

Swain, M. (2006). Verbal Protocols: What does it mean for research to use speaking as a data collection tool? In M. Chalhoub-Deville, C. A. Chapelle \& P. Duff. (Ed.), Inference and generalizability in Applied Linguistics. Multiple perspectives. Amsterdam: John Benjamins.

Tauveron, C. (1999). Comprendre et interpréter le littéraire à l'école: du texte réticent au texte proliférant. Repères, 19, 9-38.

Tauveron, C. (2004, mai). La lecture littéraire, voie possible de (ré)conciliation des élèves en difficulté avec la lecture. ÉduScol, Actes de l'université d'automne: La lecture et la culture littéraires au cycle des approfondissements. Consulté le 25 janvier 2010 dans http://eduscol.education.fr/cid46318/la-lecture-litteraire-voie-possible-de-re-conciliation-des-eleves-en-difficulte-avec-la-lecture.html

Vandendorpe, Chr. (1992). Comprendre et interpréter. In C. Préfontaine \& M. Lebrun (Éd.), La lecture et l'écriture. Enseignement et apprentissage (pp. 159-181). Montréal: Les Editions Logiques.

Mots clés: Enseignement de la lecture, allemand langue étrangère, stratégies de lecture, genres textuels, étude empirique 


\title{
Fremdsprachliches Lesen (L2) in der Schule: welche Lesestrategien für welche Textsorten?
}

\author{
Zusammenfassung \\ Der vorliegende Artikel handelt vom fremdsprachlichen Lesen (L2) in der \\ Schule und interessiert sich im Besonderen für den Einfluss, den Textsorten auf \\ die Auswahl von Lesestrategien ausüben. Die in 9. Klassen des Genfer Cycle \\ d'Orientation eingeführte didaktische Sequenz im Fach „Deutsch als Fremdspra- \\ che" hat erlaubt, den dynamischen Leseprozess beim Lösen von Aufgaben zu \\ zwei kontrastiven Textsorten (Interview und Erzähltext) anhand von Schülerin- \\ teraktionen zu analysieren. Resultate zeigen auf, dass Schüler je nach Textsorte \\ unterschiedliche Wege der Verständnissicherung angehen. Wir gehen von der \\ Annahme aus, dass diese Unterschiede mit der Funktion, die die jeweilige Text- \\ sorte ausübt, und mit den verschiedenen Kooperationsformen, die diese zwi- \\ schen Text und Leser etabliert (Eco, 1985; Ricoeur, 1983) zusammenhängt.
}

Schlagworte: Leseunterricht, Deutsch als Fremdsprache, Lesestrategien, Textgattungen, empirische Studie

\section{Leggere in lingua straniera (L2) a scuola: quali strategie di lettura per quali generi testuali?}

\section{Riassunto}

In questo articolo ci interessiamo alla lettura scolastica in lingua straniera (L2), e in particolare all'influenza del genere testuale sulla scelta delle strategie di lettura messe in atto per allievi del $9^{\circ}$ anno del Ciclo d'Orientamento del Canton Ginevra nel corso di tedesco. Il dispositivo didattico che abbiamo messo in atto ci permette di analizzare il processo di comprensione del testo come viene costruito nell'interazione tra gli allievi, nella sua dimensione dinamica e situata all'interno di consegne specifiche, usando due generi testuali in contrasto (intervista e testo narrativo). È stato possibile mettere in evidenza degli obiettivi particolari e delle traiettorie di lettura divergenti secondo il genere interessato. È possibile ipotizzare che questi risultati si spieghino attraverso il funzionamento del genere considerato, instaurando tipologie di cooperazione con il testo di natura diversa (Eco, 1985; Ricoeur, 1983).

Parole chiave: Insegnamento alla lettura, apprendimento della lettura, lingue straniere, tedesco, strategie di lettura, analisi didattica, transfer, generi testuali, dispositivo didattico, sequenza didattica, ingegneria formativa, studio empirico, analisi qualitativa, studio di caso 


\title{
Reading in a foreign language in the classroom: what kind of reading strategies for which text genre?
}

\begin{abstract}
This article deals with reading in a foreign language (L2) at school and focuses more particularly on the influence of text genre on the choice of reading strategies. We implemented a didactic sequence in 9th level German classes in Geneva that allowed us to analyse the on line reading process through students' interaction on reading tasks with two contrastive text genres (interview and narrative text). Results show that students focus on different text parts and follow different ways to construct their comprehension according to text genre. These differences can be explained by the functions of text genres which build up different types of cooperation between a reader and a text (Eco, 1985; Ricoeur, 1983).
\end{abstract}

Key words: Reading instruction, German as Foreign Language, reading strategies, text genres, empirical study 
Annexe 1

\section{Fernsehen: Videoabende}

Julian, 18 Jahre, Schüler, Berlin

\section{Sitzt du viel vor dem Fernseher?}

Ich gucke ${ }^{1}$ eigentlich gar nicht so viel Fernsehen. Aber sagen wir so: man guckt schon Fernsehen. Was man oft macht: wenn man abends nach Hause kommt oder man kommt nachts nach Hause, um drei oder vier. Dann legt man sich ins Bett und macht noch schnell den Fernseher an und guckt und zappt noch eine halbe Stunde durch die Kanäle und guckt, was gerade läuft. Da laufen meistens irgendwelche Wiederholungen vom Tag und so. Das ist ganz lustig und so. Es laufen viele Serien. Ich gucke ein paar Serien...

Welche Serien guckst du gern?

'Raumschiff Enterprise' und 'Simpsons' und so was. 'Simpsons' und... Was ich manchmal mit meinen Geschwistern zusammen gucke: so Seifenopern'2, 'Marienhof', oder 'Gute Zeiten - schlechte Zeiten' und wie sie alle heißen.

\section{Triffst du dich auch mit Freunden zum Fernsehen?}

Natürlich, wenn ein guter Film kommt, dann macht man das auch oft so: sogenannte Videoabende. Das ist vielleicht wichtig beim Fernsehen. Oft treffe ich mich mit Freunden - und das machen viele eigentlich - dann leiht man sich einen Videofilm aus oder neuerdings den DVD-Player, der viel toller sein soll, ich hab ihn leider noch nicht gesehen, und wir leihen uns also einen Videofilm aus ${ }^{3}$, setzen uns halt zusammen und gucken ein zwei Videofilme. Das ist ein Abend von 8 bis 12. Trinken dabei oder essen ein bisschen, kochen und so was alles. Das ist auf jeden Fall wichtiger als Fernsehen. Das ist lustig und macht Spaß. Meistens vier oder fünf Leute. Bei irgendeinem, der halt die Möglichkeiten dazu hat, entweder, wo die Eltern nicht zu Hause sind oder der halt die Sachen in seinem Zimmer hat.

\section{Was habt ibr da zuletzt angeguckt?}

'Matrix' haben wir angeguckt. Dann so lustige Filme wie 'Big Lebovsky', 'Fear and Loathing in Las Vegas', ja Horrorfilme haben wir uns auch mal ausgeliehen hier, 'Scream' und so was alles. Alles so Filme, die im Kino liefen, vor ein zwei Jahren. Die haben wir uns ausgeliehen.

\section{Wie wichtig ist Fernsehen oder Video für dich?}

Es ist schon wichtig. Weil, es macht schon... Was ich manchmal noch mache obwohl es eigentlich mehr so für Kinder ist - aber, ein Rollenspiel spielen. Das ist manchmal auch ganz lustig. Und Fernsehen gibt schon so 'nen gewissen Ansporn... Man sieht schon im Fernsehen viel, was man... wo man sich selber so in Situationen sieht, wo man sich selber ein bisschen vielleicht, na ja, wiederer- 
kennt und dann halt 'ne gewisse Handlungsmöglichkeit ${ }^{4}$ da drin gewinnt. Dass man weiß, wie man sich verhalten kann. Natürlich ist das nicht so wie Erfahrung 5 , nicht so viel wert, aber es ist so 'ne Sache, es macht Spaß zu gucken. So 'Enterprise' und so, wenn ich mir überlege, ich würde selbst in so einem Raumschiff sitzen - das macht Spaß zu gucken einfach. Also es ist nicht so wichtig, aber es sollte nicht fehlen.

1 gucken: sehen

2 die Seifenoper: englisch soap opera: eine sentimentale Geschichte

3 ausleihen (er lieh aus, hat ausgeliehen): etwas nehmen, es brauchen und wieder zurückgeben

4 die Handlungsmöglichkeit: etwas machen können

5 die Erfahrung: wenn man selbst etwas erlebt (leben) hat 
Annexe 2

Der Mann der nie zu spät kam

Von Paul Maar

(1)

Ich will von einem Mann erzählen, der immer sehr pünktlich war. Er hiess Wilfried Kalk und war noch nie in seinem Leben zu spät gekommen. Nie zu spät in den Kindergarten, nie zu spät zur Schule, nie zu spät zur Arbeit, nie zu spät zum Zug. Der Mann war sehr stolz darauf.

(2)

Schon als Kind war Wilfried regelmässig eine halbe Stunde vor dem Weckerklingeln aufgewacht. Wenn seine Mutter hereinkam, um ihn zu wecken, sass er angezogen in seinem Zimmer und sagte: «Guten Morgen, Mama. Wir müssen uns beeilen.» Jeden Werktag, wenn der Hausmeister in der Frühe gähnend über den Schulhof ging, um das grosse Schultor aufzuschliessen, stand Wildfried schon davor. Andere Kinder spielten nach der Schule Fussball, und schauten sich auf dem Heimweg die Schaufenster an. Das tat Wilfried nie. Er rannte sofort nach Hause, um nicht zu spät zum Essen zu kommen.

(3)

Später arbeitete Wilfried in einem grossen Büro in der Nachbarstadt. Er musste mit dem Zug zur Arbeit fahren. Trotzdem kam er nie zu spät. Er nahm den frühesten Zug und stand immer zwanzig Minuten vor der Abfahrt auf dem richtigen Bahnsteig. Kein Arbeitskollege konnte sich erinnern, dass er jemals ins Büro gekommen wäre und Wilfried Kalk nicht an seinem Schreibtisch gesessen hätte. Der Chef stellte ihn gern als gutes Beispiel hin: «Die Pünktlichkeit von Herrn Kalk, die lobe ich mir," sagte er. "Da könnte sich mancher hier eine Scheibe abschneiden.» Deswegen sagten die Arbeitskollegen oft zu Wilfried: «Könntest du nicht wenigstens einmal zu spät kommen? Nur ein einziges Mal !»

Aber Wilfried schüttelte den Kopf und sagte: «Ich verstehe nicht, welchen Vorteil es bringt, zu spät zu kommen. Ich bin mein ganzes Leben lang pünktlich gewesen.»

(4)

Wilfried verabredete sich nie mit anderen, hatte nie Termine und ging nie aus, weil er Angst hatte, zu spät zu kommen. Einmal glaubte ein Arbeitskollege, er habe Wilfried bei einer Unpünktlichkeit ertappt. Er sass im Kino und schaute sich die Sieben-Uhr-Vorstellung an. Da kam Wilfried während des Films herein und tastete sich im Dunkeln durch die Reihe. «Hallo, Wilfried! Du kommst ja zu spät», sagte der Arbeitskollege verwundert. Aber Wilfried schüttelt unwillig den Kopf und sagte: «Unsinn! Ich bin nur etwas früher gekommen, um rechtzeitig zur Neun-Uhr-Vorstellung hier zu sein». 
Ins Kino ging Wilfried sowieso sehr selten. Lieber sass er zu Hause im Sessel und studierte den Fahrplan. Er kannte nicht nur alle Ankunfts- und Abfahrtszeiten auswendig, sondern auch die Nummern der Züge und den richtigen Bahnsteig.

\section{(5)}

Als Wilfried 25 Jahre lang nie zu spät zur Arbeit gekommen war, organisierte der Chef ihm zu Ehren nach Arbeitsschluss eine Feier. Er öffnete eine Flasche Sekt und gab Wilfried ein Diplom. Es war das erste Mal, dass Wilfried Alkohol trank. Schon nach einem Glas begann er zu singen. Nach dem zweiten fing er an zu schwanken, und als der Chef ihm ein drittes Glas eingegossen hatte, mussten zwei Arbeitskollegen den betrunkenen Wilfried nach Hause bringen und ins Bett legen.

\section{(6)}

Am nächsten Morgen wachte Wilfried nicht wie üblich eine halbe Stunde vor dem Weckerklingeln auf. Als der Wecker längst geläutet hatte, schlief er immer noch tief. Er erwachte erst, als ihm die Sonne ins Gesicht schien. Entsetzt sprang er aus dem Bett, rannte zum Bahnhof. Die Bahnhofsuhr zeigte 9 Uhr 15. Viertel nach neun und er sass noch nicht hinter seinem Schreibtisch! Was würden die Kollegen sagen ? Was der Chef ? «Herr Kalk, Sie kommen zu spät, nachdem Sie erst gestern ein Diplom bekamen?!» Kopflos rannte er den Bahnsteig entlang, fiel über einen Koffer, kam zu nahe an die Bahnsteigkante, trat ins Leere und fiel hinunter auf die Schienen. Noch während des Sturzes wusste er: Alles ist zu Ende. Das ist der Bahnsteig vier, also fährt hier in diesem Moment der 9-Uhr-16 Zug ein, Zugnummer 1072, planmässige Weiterfahrt 9 Uhr 21. Ich bin tot!

\section{(7)}

Wilfried wartete fünf Sekunden, aber nichts passierte. Und weil er noch lebte, stand er auf, kletterte auf den Bahnsteig zurück und suchte einen Bahnbeamten. Als er ihn gefunden hatte, fragte er atemlos: «Der 9 Uhr 16 Zug! Was ist mit dem 9Uhr 16 Zug?» "Der hat sieben Minuten Verspätung», sagte der Beamte im Vorbeigehen. «Verspätung», wiederholte Wilfried und nickte begreifend.

(8)

An diesem Tag ging Wilfried überhaupt nicht ins Büro. Am nächsten Morgen kam er erst um 10 Uhr und am übernächsten um halb zwölf. «Sind Sie krank, Herr Kalk?» fragte der Chef erstaunt. «Nein», sagte Wilfried. «Ich habe inzwischen nur verstanden, dass Verspätungen auch sehr positiv sein können.» 
